\title{
Rendimento e qualidade de sementes de amendoim forrageiro em diferentes épocas de colheita
}

\author{
Forage Arachis seeds quality and yield as affected by harvest time
}

\author{
Luis Henrique Costa ${ }^{\mathrm{I}}$ Claudia Antonia Vieira Rossetto ${ }^{\mathrm{II}}$
}

\begin{abstract}
O objetivo do trabalho foi avaliar o rendimento $e$ a qualidade de sementes de amendoim forrageiro (Arachis pintoi) em diferentes épocas de colheita. O delineamento experimental adotado foi em blocos ao acaso, com quatro repetições e cinco tratamentos, representados pelas épocas de colheita realizadas a partir de 12 meses após a semeadura (MAS), em dezembro de 2003 e em maio de 2004, em Seropédica, RJ. Foram avaliados os componentes de produção, o rendimento de sementes com o pericarpo (vagens) e a germinação das sementes (com ou sem a remoção do pericarpo). As colheitas, realizadas no período de 16 a 18MAS, no cultivo de dezembro, e, após 12MAS, em maio, proporcionaram os maiores rendimentos de vagens de Arachis pintoi. Os parâmetros indicadores de maturidade fisiológica foram massa de 100 sementes e porcentagem de vagens soltas no solo. A remoção do pericarpo associada à temperatura alternada promoveu a superação da dormência das sementes de Arachis pintoi, após quatro meses de armazenamento.
\end{abstract}

Palavras-chave: Arachis pintoi, germinação, dormência, pericarpo, semeadura.

\section{ABSTRACT}

The aim of this study was to evaluate the forage Arachis seeds (Arachis pintoi) yield and quality as affected by time of harvesting. A completely randomized design with four replications was used. For this were carried out five harvests, starting 12 months after planting (MAP) in December of 2003 and may of 2004, in the Seropédica, RJ. The seeds pods yield, yield components and the germination of seeds with or without pod, were evaluated. The biggest production of seeds pods Arachis pintoi was obtained at 16MAP, when cultivated in December and at 12MAP, in May. The physiological maturity indicators were the weight of 100 seeds and the seeds pods percentage on the soil. The removal of pod associate the alternating temperature contributed for the overcoming of Arachis pintoi seeds dormancy, after four months of storage.

Key words: Arachis pintoi, germination, dormancy, pods, sowing.

$\mathrm{O}$ amendoim forrageiro (Arachis pintoi Krapovickas \& Gregory) apresenta insuficiente e irregular disponibilidade de sementes. A determinação do momento adequado de colheita de semente desta espécie pode tornar-se difícil devido ao hábito indeterminado de florescimento e à característica de desenvolvimento subterrâneo dos frutos, geralmente com uma semente. Assim, momentos inadequados de colheita podem prejudicar a produção e a qualidade de semente, como relatado por CARVALHO \& NAKAGAWA (2000), para várias espécies.

Para Arachis pintoi, maiores rendimentos de semente com o pericarpo (vagem) foram obtidos com a cultivar "Amarillo”, no sudoeste da Austrália, aos 12 meses após a semeadura (MAS) (COOK \& FRANKLIN, 1988), bem como com a cultivar "CIAT 18744”, no México, aos 13 MAS (ENRÍQUEZ, 2001). Já em condições brasileiras, PEÑAZOLA (1995) e PIZARRO et al. (1997) constataram que os maiores rendimentos do acesso BRA 031143 foram obtidos aos 18 e 24 meses após a instalação, respectivamente. Em relação à qualidade das sementes produzidas, segundo

IPrograma de Pós-graduação em Fitotecnia, Instituto de Agronomia, Universidade Federal Rural do Rio de Janeiro (UFRRJ), Seropédica, RJ, Brasil.

"Departamento de Fitotecnia, Instituto de Agronomia, UFRRJ, 23890-000, CP 74511, Rio de Janeiro, RJ, Brasil. E-mail: cavrosse@ufrrj.br. Autor para correspondência. 
ENRIQUEZ (2001), há possibilidade de as sementes apresentarem alto grau de dormência no momento de remoção destas, havendo, no entanto, possibilidade de superação deste fenômeno durante o período de armazenamento.

Portanto, o trabalho foi conduzido com o objetivo de avaliar o rendimento e a qualidade de sementes de Arachis pintoi em diferentes épocas de colheita. O experimento foi conduzido com Arachis pintoi na área experimental da Universidade Federal Rural do Rio de Janeiro, em Seropédica-RJ, em Planossolo, nos anos de 2003 a 2005. O delineamento experimental foi o de blocos ao acaso, com quatro repetições, sendo os tratamentos representados por cinco épocas de colheita realizadas a partir de 12 meses após a semeadura (MAS) em dezembro de 2003 (E.S.1) e em maio de 2004 (E.S.2). A densidade de semeadura foi $40 \mathrm{~kg} \mathrm{ha}^{-1}$ de sementes com o pericarpo (vagens) da cultivar “Amarillo”, com 0,5m de espaçamento entre linhas.

Foram realizadas as avaliações das porcentagens de vagens soltas no solo $\left(0,5 \mathrm{~m}^{2}\right)$, de vagens (incluindo os frutos chochos e osdeteriorados), de vagens retidas em peneira com diâmetro maior que 15/64" e de casca (pericarpo do fruto). Além disso, foi feita a determinação da massa média de 100 sementes (após a remoção do pericarpo). O rendimento de vagens, em $\mathrm{kg} \mathrm{ha}^{-1}$, foi obtido após a correção para $10 \%$ de teor de água (BRASIL, 1992). Aos 47 dias da colheita e após quatro meses de armazenamento $\left(18 \pm 2{ }^{\circ} \mathrm{C}\right.$ e $45 \pm 5 \%$ de umidade relativa do ar), quatro subamostras de 50 sementes (com ou sem o pericarpo) foram submetidas ao teste de germinação a $25^{\circ} \mathrm{C}$ e a $20-30^{\circ} \mathrm{C}$, na ausência de luz. As avaliações foram efetuadas aos cinco e aos 10 dias, com base nas recomendações para Arachis hypogaea (BRASIL, 1992). Para este teste, foi empregado o delineamento experimental inteiramente casualizado, com quatro repetições, sendo os tratamentos representados por cinco épocas de colheita realizadas a partir de 12MAS e por duas temperaturas, para os cultivos realizados em dezembro de 2003 (E.S.1) e em maio de 2004 (E.S.2). Todos os dados coletados foram submetidos à análise conjunta, reunindo as duas épocas de semeadura e as cinco épocas de colheita (GOMES, 1996). Pela análise de regressão polinomial, foram ajustadas equações, sendo que a seleção da função de melhor ajuste obedeceu ao maior coeficiente de correlação e foi complementada com a expectativa biológica.

Pode-se verificar que houve interação entre épocas de semeadura de Arachis pintoi e épocas de colheita para os parâmetros analisados (Figura 1). Na semeadura realizada em 2003 (E.S.1), a produção de vagens foi crescente desde a primeira (12MAS) até a terceira colheita (16MAS), atingindo em torno de 1000 $\mathrm{kg} \mathrm{ha-1}$, havendo a partir de então diminuição dos valores. Porém, na semeadura realizada em maio (E.S.2), houve diminuição desta produção desde a primeira (12MAS) até a penúltima colheita (18MAS). Estes resultados podem estar relacionados aos elevados valores de porcentagem de vagens formadas e de número de vagens em $1,0 \mathrm{~m}^{2}$, principalmente, para a semeadura realizada em maio. Em ambos os cultivos, estas colheitas, que proporcionaram as melhores produções de vagens, coincidiram após períodos chuvosos prolongados e elevadas temperaturas. Além disso, no cultivo de maio, entre 12 e 16MAS, houve um período com baixa precipitação pluvial. Estes resultados também foram observados por ENRIQUEZ (2001). De acordo com CRUZ et al. (1999), a maioria das leguminosas tropicais utiliza o período chuvoso para desenvolver o sistema vegetativo, produzindo os frutos somente na época seca. Após o beneficiamento das vagens colhidas nestas épocas que proporcionaram maiores rendimentos, nos dois cultivos, foram obtidos valores próximos a $30 \%$ de casca. Resultados semelhantes foram encontrados por COOK \& FRANKLIN (1988). Também foi verificado que em cada colheita, por volta de $75 \%$ das vagens desenvolveramse a $5 \mathrm{~cm}$ de profundidade do solo (Figura 1b), como constatado por FERGUSON et al. (1992).Quando foi realizada a avaliação da porcentagem de sementes soltas no solo, foram observados valores próximos a $100 \%$ aos 20MAS no cultivo de dezembro e aos 18MAS no cultivo de maio (Figura 1c). Para FERGUSON et al. (1992), valores de 97\% de vagens desprendidas da planta mãe sugerem que as sementes tenham atingido o ponto de maturidade fisiológica, devendo ocorrer, portanto, a recuperação das vagens. Além disso, em cada colheita, cerca de $95 \%$ das vagens produzidas estavam retidas em peneira com diâmetro maior que 15/ 64” Isso contrasta com Arachis hypogaea, que mantém as vagens maduras aderidas às plantas, facilitando a colheita (CARVALHO et al., 1976). Em relação à massa de 100 sementes (Figura 1d), no cultivo de dezembro, foi observado aumento dos valores da primeira (12MAS) até a última colheita (20MAS). Já no cultivo de maio, os valores permaneceram em torno de $10 \mathrm{~g}$, assim como o teor de água das sementes na colheita que esteve em torno de $50 \%$.

Após 47 dias da colheita, foi constatada ausência de germinação das sementes, que apresentavam $8 \%$ de água, provavelmente devido à dormência, como constatado também por FERGUSON (1994), FLOR (1998) e ENRIQUEZ (2001). No entanto, após quatro meses de armazenamento, foram 


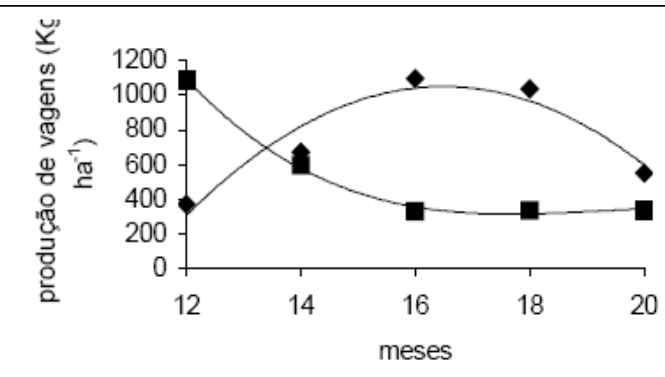

$\bullet$ E.S.1 $\square$ E.S.2

A

(•) $\mathrm{y}=-9000,51+1218,87 \mathrm{x}-36,97 \mathrm{x}^{2}$

(घ) $y=+7594,20-816,72 x+22,76 x^{2}$

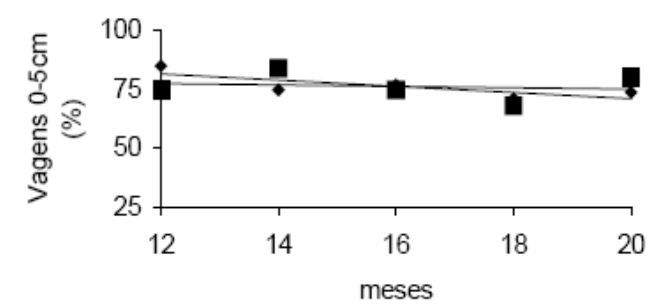

- E.S.1 घE.S.2

B

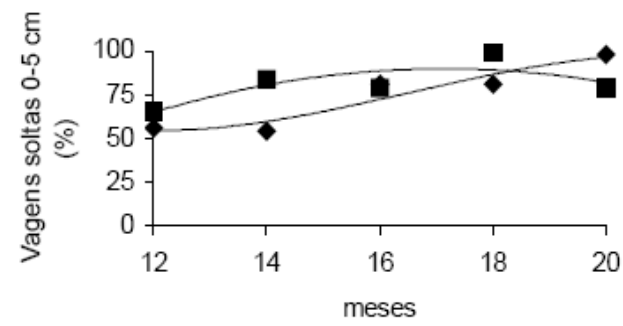

- E.S.1 घE.S.2

$\mathrm{C}$

(•) $\mathrm{y}=+518,71-95,03 \mathrm{x}+6,19 \mathrm{x}^{2}-0,12 \mathrm{x} \quad \mathrm{R}^{2}=0,89 * *$

(a) $y=-187,91+32,43 x-0,94 x^{2} \quad R^{2}=0,64$ *

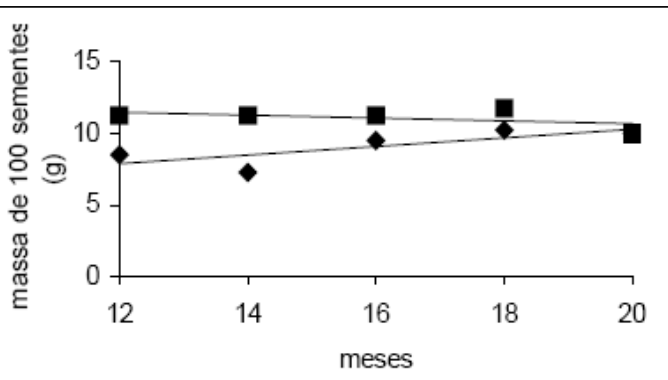

- E.S.1 שE.S.2

D

(•) $\mathrm{y}=+4,3+0,3 \times \mathrm{R}^{2}=0,92^{* * *}$

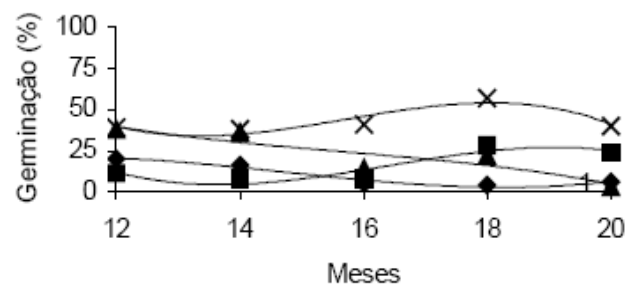

1-E.S.1 घ 2-E.S.1 \ 3-E.S.1 × 4-E.S.1

$\mathrm{E}$

(•) $y=-264,37+64,01 x-4,60 x^{2}+0,10 x^{3} \quad R^{2}=0,96$ ** (-) $\mathrm{y}=+1149,50-220,16 \mathrm{x}+13,82 \mathrm{x}^{2}-0,28 \mathrm{x}^{3} \mathrm{R}^{2}=0,85^{*}$ *

(•) $\mathrm{y}=+354,89-56,92 \mathrm{x}+3,42 \mathrm{x}^{2}-0,07 \mathrm{x}^{3} \quad \mathrm{R}^{2}=0,81^{\text {*** }}$

(x) $y=+436,90-278,85 x+18,16 x^{2}-0,38 x^{3} R^{2}=0,82$ **

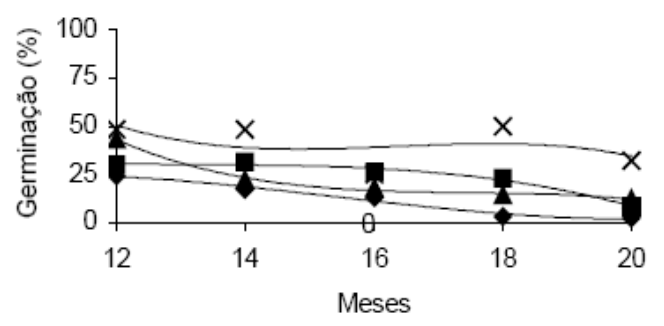

• 1-E.S.2 • 2-E.S.2 ^ 3-E.S.2 × 4-E.S.2

F

(•) $y=-157,63+40,82 x-2,89 x^{2}+0,06 x^{3} R^{2}=0,97 * *$ (a) $y=+174,74-32,60 x+2,46 x^{2}-0,06 x^{3} R^{2}=0,98$ * *

(•) $\mathrm{y}=+852,09+143,09 \mathrm{x}+8,17 \mathrm{x}^{2}-0,15 \mathrm{x}^{3} \mathrm{R} 2=0,99$ * *

(x) $y=-0,20 x^{3}+10,21 x^{2}-165,52 x+925,74 R^{2}=0,47 *$

Figura 1 - Produção de vagens (a), porcentagem de vagens a $5 \mathrm{~cm}$ de profundidade no solo (b), porcentagem de vagens soltas da planta mãe (c), massa de 100 sementes (d) e germinação de sementes de Arachis pintoi, colhidas no cultivo de dezembro de 2003 (e) e de maio de 2004 (f), após quatro meses de armazenamento a $25^{\circ} \mathrm{C}$, com (1) e sem pericarpo (2) e a $20-30^{\circ} \mathrm{C}$, com (3) e sem pericarpo (4). Avaliações realizadas após 12, 14, 16, 18, e 20 meses da semeadura. Seropédica, RJ. 
constatadas as maiores porcentagens de germinação, provavelmente devido à redução de sementes nãogerminadas (duras, mortas e dormentes), provenientes da amostra obtida aos 18MAS no cultivo de dezembro de 2003, principalmente após a remoção do pericarpo da vagem associada à temperatura de $20-30^{\circ} \mathrm{C}$ (Figura 1e), sugerindo que a dormência não é devida somente à impermeabilidade do pericarpo das vagens, como também verificado por FERGURSON (1994). Além disso, resultados semelhantes foram observados na avaliação da amostra obtida aos 18MAS, proveniente do cultivo em maio (Figura 1f). Porém, estas sementes não atendem ao padrão de germinação, que é de $60 \%$ (BRASIL, 1999). Estes resultados podem estar relacionados com a porcentagem de vagens soltas no solo (Figura 1c) e com a massa de 100 das sementes (Figura 1d). Resultados semelhantes foram obtidos por FERGUSON et al. (1992), que verificaram maiores valores de massa seca de sementes e de viabilidade na amostra de sementes desprendidas da planta mãe, caracterizando estes parâmetros como indicadores de maturidade fisiológica.

Conclui-se que as colheitas, realizadas no período de 16 a 18 meses após a semeadura no cultivo de dezembro e após 12 meses da semeadura em maio proporcionaram os maiores rendimentos de vagens de Arachis pintoi. Os parâmetros indicadores de maturidade fisiológica foram massa de 100 sementes e porcentagem de vagens soltas no solo. A remoção do pericarpo associada à temperatura alternada promoveu a superação da dormência das sementes de Arachis pintoi após quatro meses de armazenamento.

\section{AGRADECIMENTO}

Ao Conselho Nacional de Desenvolvimento Científico e Tecnológico (CNPq), pela Bolsa de Produtividade de Pesquisa ao autor C.A.V. Rossetto.

\section{REFERÊNCIAS}

BRASIL. Ministério da Agricultura e da Reforma Agrária. Regras para análise de sementes. Brasilia: SNAD/DNPV/ CLAV, 1992. 385p.
BRASIL. Ministério da Agricultura. Comissão Estadual de Sementes e Mudas. Padrão de sementes para a safra 99/ 2000. Campinas: Secretaria de Agricultura do Estado de São Paulo, 1999. 11p.

CARVALHO, N.M.; NAKAGAWA, J. Sementes: ciência, tecnologia e produção. 4.ed. Jaboticabal: Funep, 2000. 588p.

CARVALHO, N.M. et al. Maturação de sementes de amendoim (Arachis hypogaea L.). Científica, v.4, n.1, p.39-42, 1976.

COOK, B.G.; FRANKLIN, T.G. Crop management and seed harvesting of Arachis pintoi Krap. \& Greg. Journal of Applied Seed Prodution, v.6, p.26-30, 1988.

CRUZ, E.D. et al. Produção de sementes de A. pintoi Krap. \& Greg. na Amazônia Oriental Brasileira. Pasturas Tropicales, v.21, n.3, p.59-61, 1999.

ENRÍQUEZ Q.J.F. Efecto de la aplicación de cal y la época de cosecha en la producción de semilla de Arachis pintoi CIAT 18744. Pasturas Tropicales, v.23, n.1, p.25-28, 2001.

FERGUSON, J.E. Seed biology and seed systems for Arachis pintoi. In: KERRIDGE, P.C.; HARDY, B. (Eds). Biology and agronomy of forage Arachis. Cali: CIAT, 1994. Cap.11, p.122-133.

FERGUSON, J.E. et al. Avances y perspectivas em la producción de semilla de Arachis pintoi. Pasturas Tropicales, v.14, n 2, p.14-22, 1992.

FLOR, C.A. Caracterización de la calidade fisiológica de la semilla de Arachis pintoi Krap. et Greg. nom. nud. en tres ambientes de almacenamiento. Acta Agronomica, v.47, n.2, p.9-19, 1998.

GOMES, J.L.P. Curso de estatística experimental. São Paulo: Nobel, 1996. 404p.

PEÑALOZA, A.D.P.S. Germinação de sementes de Arachis pintoi obtidas em condições distintas de multiplicação. In: REUNIÃO BRASILEIRA DE ZOOTECNIA, 32., Brasília, DF. Anais... Brasília: SBT, 1995. p.78-79.

PIZARRO, E.A. et al. Producción y persistencia de siete accesiones de Arachis pintoi associadas com Paspalum maritimum en el Cerrado brasileño. Pasturas tropicales, v.19, n.2, p.40-44, 1997. 